\title{
CONSTRUCCIÓN DEL SÍ MISMO Y CATEGORIZACIÓN DE LOS SIGNIFICADOS PERSONALES DE ACUERDO CON LAS FORTALEZAS DEL CARÁCTER: ESTUDIO COMPARATIVO EN PACIENTES CON TRASTORNOS DEPRESIVOS SELF CONSTRUCTION AND CATEGORIZATION OF PERSONAL MEANINGS ACCORDING TO THE CHARACTER STRENGTHS: COMPARATIVE STUDY IN PATIENTS WITH DEPRESSIVE DISORDERS
}

\author{
LuIS ÁNGEL SAÚL \\ M. ÁNGELES LóPEZ-GONZÁLEZ \\ Fernando Rubio-Garay \\ Marcela Paz González-Brignardello \\ Departamento de Personalidad, Evaluación y Tratamientos Psicológicos. \\ Facultad de Psicología, UNED \\ lasaul@psi.uned.es
}

Cómo referenciar este artículo/How to reference this article:

Saúl, L. A., López-González, M. A., Rubio-Garay, F. y González-Brignardello, M. P. (2012). Construcción del sí mismo y categorización de los significados personales de acuerdo con las fortalezas del carácter: estudio comparativo en pacientes con trastornos depresivos. Acción Psicológica, 9(2), 3-20 doi: http://dx.doi.org/10.5944/ap.9.2.4100

\section{Resumen}

El objetivo de este estudio fue explorar la autodefinición y la diferenciación del sí mismo en un grupo de pacientes con trastornos depresivos $(\mathrm{n}=30)$ comparándolo con una muestra comunitaria $(\mathrm{n}=30)$. Los significados personales de estos sujetos, elicitados a través de la Técnica de la Rejilla, se categorizaron según las virtudes y las fortalezas del carácter de Peterson y Seligman (2004) y se analizó la posición que las personas otorgan a los elementos «yo actual»y «yo ideal». Además, se estudió la diferenciación del sí mismo a través del análisis de diferentes índices de medida cognitiva que evalúan la autoestima, el aislamiento social autopercibido y la adecuación percibida en los otros. Los resultados más destacables muestran que las personas con depresión presentan más constructos en las categorías autocontrol y autorregulación e inteligencia emocional, personal y social que la muestra comunitaria. Asimismo, se encontraron diferencias significativas entre los dos 
grupos en la posición del «yo actual» en relación al «yo ideal». En este sentido, el grupo clínico elicita un mayor número de constructos discrepantes en Coraje, Sabiduría, Transcendencia, Moderación, Humanidad y Justicia, y el grupo no clínico presenta más constructos congruentes en todas las virtudes menos en Justicia. En cuanto a los constructos dilemáticos, en el grupo clínico se observan más constructos en Coraje y Moderación. Finalmente, los pacientes con depresión presentan una baja autoestima, una construcción negativa del yo y se perciben diferentes con respecto a su entorno social. Estos datos, tomados en conjunto con la correlación positiva encontrada entre el «yo ideal» y los «otros» (adecuación percibida en los otros), indicaría un perfil de aislamiento en la muestra clínica.

Palabras clave: constructivismo, sí mismo, análisis de contenido, constructos personales, técnica de rejilla, fortaleza del carácter, depresión.

\section{Abstract}

The aim of this study was to explore the selfdefinition and self-differentiation of a group of patients with depressive disorders $(n=30)$ compared with a community sample $(\mathrm{n}=30)$. Personal meanings of these subjects, elicited by Grid Technique, were categorized according to the virtues and character strengths of Peterson and Seligman (2004) and analyzed the position that people attach to the elements "present self» and «ideal self». In addition, it was studied the self-differentiation through the analysis of several indices that assess self-esteem, perceived social isolation and perceived adequacy in the others. The main results show that people with depression have more constructs in self-control, self-regulation and emotional, personal and social intelligence categories than community sample. Furthermore, significant differences between the two groups were found in the position of "present self» in relation to «ideal self». In this sense, the clinical group elicits more discrepant constructs in Courage, Wisdom, Transcendence, Moderation, Humanity and Justice, and the nonclinical group presents more congruent constructs in all virtues least Justice. Re- garding dilemmatic constructs, more of them are showed in Moderation and Courage categories in the clinical group. Finally, patients with depression show low self-esteem and they perceive themselves different with respect to their social environment. These data, taken together with the positive correlation between the «ideal self» and "others» (perceived adequacy in the others), indicate a tentative profile of isolation in the clinical sample.

Keywords: constructivism, self, content analysis, personal constructs, repertory grid technique, character strengths, depression.

\section{Introducción}

Desde un enfoque epistemológico constructivista se asume que el ser humano construye activamente el conocimiento y da sentido a la realidad a partir de su propia experiencia. La Psicología de los Constructos Personales (PCP) estudia el mundo de los significados que cada persona elabora para predecir los acontecimientos y otorgarles un sentido (Botella y Feixas, 1998). Para Kelly (1955/1991), un constructo personal es una dimensión de significado que implica la captación de una diferencia, puesto que el significado de algo (e.g., valiente) comprende siempre un contraste (e.g., cobarde). Cada persona emplea su propio sistema de constructos personales que, a modo de guía, le sirve para establecer semejanzas y diferencias entre distintos elementos. De esta manera, cada persona elige aquel polo del constructo que le es más útil para anticipar los acontecimientos de manera coherente a su propia experiencia. Una construcción sin contraste sería una estructura totalmente homogénea e indiferenciada y, del mismo modo, una estructura sin semejanzas constituiría una heterogeneidad caótica sin puntos de referencia en los que apoyarse.

La exploración del sistema de constructos de una persona se ha llevado a cabo fundamentalmente mediante la Técnica de la Rejilla (Kelly, 1955). Se trata de un instrumento que evalúa las dimensiones y la estructura del significado personal (Feixas y Botella, 2003; 
Feixas y Cornejo, 1996), por lo que puede considerarse una herramienta de evaluación eminentemente idiográfica. No obstante, en el campo de la investigación, resulta de interés el poder realizar comparaciones intersujetos desde una aproximación también nomotética. Mediante esta técnica se han venido desarrollando diversos índices generales para el estudio de la estructura cognitiva (véase Feixas y Cornejo, 1996 para una revisión); sin embargo, el análisis del contenido de los constructos elicitados ha recibido una menor atención por parte de los investigadores. Para abordar ese cometido, se requieren sistemas de categorización de significados que permitan el contraste y la comparación, si bien, desde la PCP no se ha producido el desarrollo deseado, ni en el estudio del análisis de contenido de los constructos personales ni en la elaboración de sistemas clasificatorios. La primera propuesta surgió de la mano de Landfield (1971) quien creó una clasificación basada en 32 categorías. Sin embargo, este sistema ha sido criticado porque sus categorías no son exhaustivas ni mutuamente excluyentes, y porque mezcla aspectos formales y de contenido (Feixas, Geldchläger, Carmona y Garzón, 2002; Montesano, Feixas y Varlotta, 2009). En la última década, el grupo de Feixas (Feixas et al., 2002, Feixas, Saúl y Ávila-Espada, 2009) ha creado otra propuesta de clasificación, el Sistema de Categorías para Constructos Personales (SCCP), formado por 45 categorías que se corresponden con seis áreas: moral, emocional, relacional, personal, intelectual y de valores/intereses.

Junto a estos dos sistemas de categorización utilizados en la investigación, otro método de análisis del contenido de los constructos podría basarse en la clasificación sistemática realizada por el equipo de investigación de Seligman (Peterson y Seligman, 2004; Seligman, 2003) dentro del marco de la Psicología Positiva. Este nuevo enfoque de la psicología persigue analizar e identificar los aspectos relacionados con la felicidad y el bienestar, incluyendo el rol que las fortalezas, las virtudes personales (Seligman y Peterson, 2004) y los sistemas sociales juegan en su promoción (Carr, 2004). Consiste en una visión complementaria al modelo psicológico clásico basado en el trastorno, el desajuste y el déficit, proponiendo un marco teórico que explique las condiciones y procesos que contribuyen al funcionamiento óptimo de las personas, los grupos y las instituciones (Gable y Haidt, 2005). En la búsqueda de un lenguaje común, Seligman y su equipo buscaron virtudes y fortalezas universales reconocidas en todas las culturas mediante el análisis de textos religiosos y filosóficos antiguos (véase Peterson y Seligman, 2004). Como resultado de su estudio histórico y cultural consensuan seis grandes virtudes (virtudes básicas), que «son definitivamente ubicuas y probablemente universales» (Carr, 2004, p. 53): Sabiduría, Coraje, Humanidad, Justicia, Moderación o Templanza y Trascendencia. Cada una de estas virtudes se compone de 24 rasgos más específicos a las que denominaron fortalezas del carácter (ver Apéndice A).

El estudio de los significados personales ha suscitado interés tanto en el campo teórico como aplicado en el área de la salud mental. En el caso específico de la depresión, en los últimos tiempos se han llevado a cabo diversos estudios en los que se ha utilizado la Técnica de la Rejilla (e.g., De Bonis, 1998; Feixas, Erazo-Caicedo, Harter y Bach 2008; Hanieh y Walker, 2007; Sanz, 1991, 1992). En líneas generales, las investigaciones realizadas muestran que la entramada red de constructos en estas personas con depresión se caracteriza por una autoconstrucción negativa y por la anticipación fatalista del futuro (Kelly 1955/1991). Esta autoconstrucción negativa implica una fuerte auto-devaluación personal que se traduce en valoraciones más negativas y menos positivas del yo real, en una mayor discrepancia entre la construcción de los elementos «yo real» y «yo ideal» (Sanz, 1991) y en el empleo de un número reducido de constructos para describirse a sí mismo y a los demás (Feixas et al., 2008). En este sentido, la presencia de constructos cuyo contenido revela una fuerte minusvaloración junto con una visión negativa de sí mismo y de los otros (perfil de negatividad o de pesimismo) es característico de las personas con depresión (Feixas y Cornejo, 1996). Además, según Kelly (1955/1991) la depresión va unida a una gradual constricción o estrechamiento del campo perceptivo de la persona. Esta constricción le permite postergar la 
revisión de su sistema de constructos, evitando así situaciones ansiógenas o impredecibles, esto es, permitiéndole minimizar la invalidación personal (Feixas et al., 2008; Sanz, 1991).

Las fortalezas del carácter están directamente relacionadas con la consecución del bienestar, la felicidad y la validación personal, y en las personas con depresión, es posible que estas fortalezas se contrapongan al sesgo y a la tríada cognitiva negativa (Beck, Rush, Shaw y Emery, 1983/2000). Teniendo en cuenta estas premisas, en este trabajo nos proponemos explorar estas fortalezas a partir de los constructos personales elicitados a través de la TR. Para ello, cada constructo personal se clasifica como una única unidad de significado en su categoría más adecuada y se recoge la valencia que le asigna el sujeto a dicho significado, en función de cómo se construye a sí mismo. En este sistema de categorización no solo se clasifica cada constructo personal como una única unidad de significado en su categoría más adecuada, sino que se recoge la valencia que le asigna el sujeto a dicho significado, en función de cómo se construye a sí mismo. Desde este planteamiento, el objetivo de este estudio es explorar la construcción del sí mismo en un grupo de personas con trastornos depresivos frente a otro grupo sin patología, y se concreta en dos objetivos específicos: (a) analizar la autodefinición del sí mismo desde el estudio de la posición de los elementos «yo actual» y el «yo ideal», previa categorización sistemática de los constructos personales, y (b) estudiar la diferenciación del sí mismo en cuanto a la autoestima, el aislamiento social autopercibido y la adecuación percibida en los otros.

\section{Método}

\section{Participantes}

En la investigación participaron 60 personas (17 hombres y 43 mujeres) con edades comprendidas entre los 19 y 61 años de edad. La muestra se obtuvo mediante muestreo no probabilístico accidental.
El grupo clínico estuvo formado por 30 participantes $(16.6 \%$ hombres y $83.3 \%$ mujeres) con un rango de edad entre 19 y 50 años ( $X=26.17, D T=7.76)$. Los criterios de inclusión del grupo clínico fueron: (a) personas demandantes de ayuda psicológica en un servicio de atención psicológica universitaria; y (b) cumplimiento de los criterios diagnósticos de algún Trastorno del Estado de Ánimo según el DSM-IV-TR (APA, 2000): Trastorno Depresivo Mayor, Episodio Único (n = 21), Trastorno Depresivo Mayor, Recidivante, Moderado $(\mathrm{n}=1)$, Trastorno Depresivo Mayor, Recidivante, Grave, Sin Síntomas Psicóticos $(\mathrm{n}=1)$, Trastorno Distímico $(\mathrm{n}=1)$ y Trastorno Depresivo No Especificado $(\mathrm{n}=6)$. Se excluyeron aquellas personas que presentaban historia psiquiátrica previa, uso/abuso de sustancias, condición médica inestable, retraso mental y/o cualquier otro trastorno generalizado del desarrollo.

El grupo no clínico estuvo compuesto por 30 participantes (40\% hombres y 60\% mujeres), todos ellos voluntarios reclutados en sus ambientes naturales, no sometidos a ninguna clase de terapia. El rango de edad estuvo comprendido entre los 20 y los 61 años $(\bar{X}=37.50, D T=9.81)$.

\section{Instrumentos de evaluación}

Técnica de la Rejilla (Kelly, 1955)

La técnica de la Rejilla (Kelly, 1955) es un instrumento que pretende evaluar cómo las personas dan sentido a su experiencia. Tiene formato de cuadrícula y está formada por columnas y por filas que se corresponden con los elementos y los constructos, respectivamente. De entre las diversas modalidades de diseño de la técnica se ha escogido la Técnica de Rejilla Interpersonal, en la que se utilizan como elementos personas significativas, incluyendo una «persona non grata» y como elementos del sí mismo el «yo actual»y el «yo ideal».

Los constructos son ideas o pensamientos que la persona tiene acerca de los elementos (Marcet, 1985), tienen un carácter bipolar y para obtenerlos se formulan preguntas de similaridad o contraste entre pares de elementos 
(elicitación diádica). A continuación se procede a puntuar cada uno de los elementos y para ello se calibra cada uno de los polos de los constructos en un sistema de puntuación de intervalo. Se asigna la siguiente puntuación: 1 (muy), 2 (bastante) y 3 (un poco) al primer polo o emergente, 4 al punto medio, y 5 (un poco), 6 (bastante) y 7 (muy) al polo de contraste.

De la TR se ha derivado una gran variedad de índices y medidas (para una revisión ver Feixas y Cornejo, 1996). En este estudio se han empleado las siguientes:

1. Relacionadas con la posición de los elementos «yo actual» $y$ «yo ideal». Se puede establecer la siguiente tipología: (a) constructos congruentes, aquellos que no se diferencian más de un punto entre el "yo actual» y el "yo ideal» en la escala de 7 puntos e indican los constructos en los que la persona no desea cambiar; (b) constructos discrepantes, constructos en los que existe una gran diferencia entre el «yo actual»y el "yo ideal», reflejada por una distancia de al menos 4 puntos; (c) constructos dilemáticos, constructos que no ofrecen un curso claro de acción, ya que sitúan al ideal en una posición intermedia entre los dos polos que definen el constructo (puntuación de 4 en el elemento «yo ideal»); y (d) categoría indefinida, son aquellos que no encajan en ninguna de las categorías anteriores, esto es, la distancia entre el «yo actual» y el «yo ideal» se sitúa entre 2 y 4 puntos, siempre que el «yo ideal» no presente una puntuación de 4 .

2. Diferenciación del sí mismo (véase Feixas y Cornejo, 1996): (a) correlación entre los elementos "yo actual» - "yo ideal», considerada como una medida de autoestima; (b) correlación "yo actual» - «otros», se calcula a partir de la correlación entre el elemento "yo actual» y un elemento "artificial», resultado de promediar todos los elementos menos el «yo actual» y el "yo ideal», y sugiere el aislamiento social autopercibido, indicativo de la soledad del sujeto; y (e) correlación «yo ideal» - "otros», calculado mediante la correlación entre los elementos «yo ideal» y el elemento artificial «otros», y es un indicador de la adecuación percibida en los otros.

\section{Procedimiento}

La muestra se obtuvo de la Unidad de Atención Psicológica y Salud Mental para el Universitario de la Universidad de Salamanca, centro integrado dentro del Proyecto Multicéntrico Dilema (Feixas y Saúl, 2004; véase www.usal.es/tcp). La evaluación de la muestra clínica se llevó a cabo en dos sesiones; en la primera sesión, se realizó una anamnesis y recogida de datos sociodemográficos, y se estableció el diagnóstico según los criterios del Manual Diagnóstico y Estadístico de los Trastornos Mentales (4. ${ }^{a}$ Ed., rev.; DSM-IVTR; Americam Psychiatric Association [APA], 2000). En una segunda sesión, a los sujetos diagnosticados de un trastorno del estado de ánimo, se les administró la TR y el Beck Depression Inventory (BDI- I; Beck, Rush, Shaw, Emery, 1983/2000). Solo se incluyeron en este estudio los sujetos con una puntuación igual o superior a 18 en el BDI-I.

La muestra no clínica se extrajo de voluntarios en sus ambientes naturales que fueron evaluados por estudiantes de psicología de la UNED tras ser entrenados en el manejo de la TR. Previamente a su administración, los participantes cumplimentaron el instrumento de cribado psicopatológico Symptom Check List90-R (SCL-90-R; Derogatis, 1994, versión española de González de Rivera, de las Cuevas, Rodríguez y Rodríguez, 2002) y se consideraron en el estudio personas con puntuaciones por debajo del percentil 80 y con valores en el Índice de Severidad Global inferiores a 0.63 en hombres y a 0.77 en mujeres.

Para el análisis del contenido de los constructos personales se procedió a la codificación de cada uno de ellos como una unidad de significado acotado por dos polos (Kelly, 1955/1991) y de acuerdo con el sistema de las virtudes y las fortalezas del carácter de Peterson y Seligman (2004). Así, cada categoría conformaba un eje de coordenadas sobre el que se clasificó cada constructo dependiendo del ámbito de pertenencia a una determinada dimensión. Las discrepancias surgidas durante el proceso de codificación se resolvieron por consenso entre expertos. En la categoría Sabiduría se englobaron aquellos constructos relaciona- 
dos con el conocimiento (i.e. culto vs. inculto, curioso vs. sin interés por el mundo); en Coraje, aquellos que trataban sobre la consecución o no de metas ante situaciones de dificultad externa o interna (i.e. valiente vs. cobarde, con tesón vs. apagado); en Humanidad comprendían aspectos sobre las relaciones interpersonales (i.e., egoísta vs. generoso, simpático vs. antipático); en Justicia se incluyeron significados sobre aspectos (in)cívicos en la vida en comunidad (i.e. justo vs. injusto, leal vs. desleal); en Moderación se recogieron constructos relacionados con la (falta de) autorregulación o la (falta de) motivación (i.e. prudente vs. imprudente, modesto vs. ostentoso) y, por último en Trascendencia aquellos constructos elicitados que tenían que ver con la conexión del individuo con el universo y proveían de significado a su vida (vs. optimista vs. pesimista, con esperanza vs sin esperanza). Además de la categorización del contenido de los constructos, para cada uno de ellos se determinó la posición de los elementos «yo actual» y «yo ideal» a través de las puntuaciones otorgadas por los participantes.

\section{Diseño y variables}

Se trata de un diseño ex post facto prospectivo simple. La variable independiente fue la condición clínica (presencia/ausencia de trastorno del estado del ánimo). Las variables dependientes consideradas fueron: (a) como medidas de autodefinición del sí mismo, por una parte, cada una de las seis virtudes o de las 24 fortalezas del carácter operativizadas a través del número de constructos previamente asignados en cada una de ellas, y, por otra parte, para conocer la posición de los elementos "yo actual» - "yo ideal, el número o porcentaje de constructos congruentes, discrepantes, dilemáticos o indefinidos en cada rejilla; (b) como medidas de diferenciación del sí mismo, la autoestima, el aislamiento social autopercibido y la adecuación percibida en los otros, a través del estudio de las correlaciones "yo actual» - «yo ideal», «yo actual» - «otros» y «yo ideal»-«otros».

\section{Análisis de datos}

Los datos obtenidos a partir de las rejillas se analizaron utilizando el programa RECORD v 4.0 (Feixas y Cornejo, 2002) y el paquete informático SPSS ${ }^{\circledR}$ para Windows (Statistical Package for Social Sciences), versión 19.01.

La relación entre las variables categóricas se estudió mediante tablas de contingencia y estadísticos no paramétricos (Chi cuadrado y coeficiente de contingencia) y se completó con análisis de residuos tipificados corregidos, considerándose como estadísticamente significativos aquellos mayores o iguales de |1.96|, con un nivel de significación de un 95\%.

La posición de los elementos «yo actual»y «yo ideal» y la diferenciación del sí mismo se realizó mediante sendos análisis multivariados de la varianza (MANOVA). Previamente se comprobó que no se incumplieran gravemente los supuestos de normalidad multivariada, linealidad, homogeneidad de las matrices de varianzas-covarianzas e independencia de las observaciones.

\section{Resultados}

\section{Autodefinición del sí mismo}

\section{Análisis de contenido}

El número de constructos elicitados por toda la muestra ascendió a 1030 (562 en el grupo no clínico y 468 en el grupo clínico) y se distribuyeron en las virtudes de la siguiente forma: Humanidad (29.50\%), Coraje (24.47\%), Moderación (19.03\%), Sabiduría (13.11\%), Transcendencia $(10.49 \%)$ y Justicia $(3.40 \%)$.

El estudio de la relación entre las variables condición clínica (presencia/ausencia de trastorno del estado de ánimo) y virtudes mediante la prueba de Chi-cuadrado puso de manifiesto que ambas variables eran independientes: $X^{2}(5)=7.362$, $p=0.195$. Posteriormente, se analizó la relación entre las variables condición clínica y fortalezas del carácter. En este caso, la prueba Chi cuadrado mostró que ambas variables estaban relacionadas: $X^{2}(22)=72.383, p=0.000$. El análisis de residuos tipificados puede verse en la tabla 1. 
Tabla 1

Tabla de contingencia entre las 24 fortalezas del carácter y la condición clínica

\begin{tabular}{|c|c|c|c|c|c|c|c|c|c|c|c|}
\hline & & Clínica & No clínica & & & Clínica & No clínica & & & Clínica & No clínica \\
\hline \multirow[t]{2}{*}{1} & $\begin{array}{c}\text { Rec. } \\
\%\end{array}$ & $\begin{array}{c}20 \\
55.60 \%\end{array}$ & $\begin{array}{c}16 \\
44.40 \%\end{array}$ & 9 & $\begin{array}{c}\text { Rec. } \\
\%\end{array}$ & $\begin{array}{c}23 \\
69.70 \%\end{array}$ & $\begin{array}{c}10 \\
30.30 \%\end{array}$ & 17 & $\begin{array}{c}\text { Rec. } \\
\%\end{array}$ & $\begin{array}{c}9 \\
37.50 \%\end{array}$ & $\begin{array}{c}15 \\
62.50 \%\end{array}$ \\
\hline & RTC & 1.2 & -1.2 & & RTC & 2.8 & -2.8 & & RTC & -0.8 & 0.8 \\
\hline \multirow[t]{3}{*}{2} & Rec. & 1 & 2 & 10 & Rec. & 31 & 49 & 18 & Rec. & 8 & 20 \\
\hline & $\%$ & $33.30 \%$ & $66.70 \%$ & & $\%$ & $38.80 \%$ & $61.30 \%$ & & $\%$ & $28.60 \%$ & $71.40 \%$ \\
\hline & RTC & -0.4 & 0.4 & & RTC & -1.3 & 1.3 & & RTC & -1.8 & 1.8 \\
\hline \multirow[t]{3}{*}{3} & Rec. & 20 & 41 & 11 & Rec. & 38 & 59 & 19 & Rec. & 76 & 62 \\
\hline & $\%$ & $32.80 \%$ & $67.20 \%$ & & $\%$ & $39.20 \%$ & $60.80 \%$ & & $\%$ & $55.10 \%$ & $44.90 \%$ \\
\hline & RTC & -2 & 2 & & RTC & -1.3 & 1.3 & & RTC & 2.4 & -2.4 \\
\hline \multirow[t]{3}{*}{4} & Rec. & 11 & 19 & 12 & Rec. & 78 & 49 & 20 & Rec. & 4 & 3 \\
\hline & $\%$ & $36.70 \%$ & $63.30 \%$ & & $\%$ & $61.40 \%$ & $38.60 \%$ & & $\%$ & $57.10 \%$ & $42.90 \%$ \\
\hline & RTC & -1 & 1 & & RTC & 3.9 & -3.9 & & RTC & 0.6 & -0.6 \\
\hline \multirow[t]{3}{*}{5} & Rec. & 2 & 3 & 13 & Rec. & 2 & 5 & 21 & Rec. & 0 & 0 \\
\hline & $\%$ & $40.00 \%$ & $60.00 \%$ & & $\%$ & $28.60 \%$ & $71.40 \%$ & & $\%$ & $0 \%$ & $0 \%$ \\
\hline & RTC & -0.2 & 0.2 & & RTC & -0.9 & 0.9 & & RTC & 0 & 0 \\
\hline \multirow[t]{3}{*}{6} & Rec. & 16 & 12 & 14 & Rec. & 10 & 13 & 22 & Rec. & 31 & 24 \\
\hline & $\%$ & $57.10 \%$ & $42.90 \%$ & & $\%$ & $43.50 \%$ & $56.50 \%$ & & $\%$ & $56.40 \%$ & $43.60 \%$ \\
\hline & RTC & 1.3 & -1.3 & & RTC & -0.2 & 0.2 & & RTC & 1.7 & -1.7 \\
\hline \multirow[t]{3}{*}{7} & Rec. & 37 & 89 & 15 & Rec. & 2 & 3 & 23 & Rec. & 10 & 27 \\
\hline & $\%$ & $29.40 \%$ & $70.60 \%$ & & $\%$ & $40.00 \%$ & $60.00 \%$ & & $\%$ & $27.00 \%$ & $73.00 \%$ \\
\hline & RTC & -3.9 & 3.9 & & RTC & -0.2 & 0.2 & & RTC & -2.3 & 2.3 \\
\hline \multirow[t]{3}{*}{8} & Rec. & 27 & 38 & 16 & Rec. & 6 & 0 & 24 & Rec. & 6 & 3 \\
\hline & $\%$ & $41.50 \%$ & $58.50 \%$ & & $\%$ & $100.00 \%$ & $0.00 \%$ & & $\%$ & $66.70 \%$ & $33.30 \%$ \\
\hline & RTC & -0.7 & 0.7 & & RTC & 2.7 & -2.7 & & RTC & 1.3 & -1.3 \\
\hline
\end{tabular}

Nota. Rec. (recuento), \% (frecuencia dentro de cada fila), RTC (Residuos Tipificados Corregidos), 1 (curiosidad, interés por el mundo), 2 (amor por el conocimiento y el aprendizaje), 3 (juicio, pensamiento crítico, mentalidad abierta), 4 (ingenio, originalidad e inteligencia práctica), 5 (perspectiva), 6 (valentía), 7 (perseverancia y diligencia), 8 (integridad, honestidad y autenticidad), 9 (vitalidad por las cosas), 10 (amor, apego, capacidad de amar y ser amado), 11 (simpatía, amabilidad y generosidad), 12 (inteligencia emocional, personal y social), 13 (ciudadanía, civismo, lealtad y trabajo en equipo), 14 (sentido de la justicia, equidad), 15 (liderazgo), 16 (capacidad de perdonar, misericordia), 17 (modestia o humildad), 18 (prudencia, discreción o cautela), 19 (autocontrol o autorregulación), 20 (apreciación de la belleza y la excelencia, capacidad de asombro), 21 (gratitud), 22 (esperanza, optimismo, proyección hacia el futuro), 23 (sentido del humor), 24 (espiritualidad, fe y sentido religioso).

Las celdas sombreadas señalan las fortalezas más elicitadas por toda la muestra.

Tabla 2

Medias, desviaciones típicas y significación estadística de los índices relacionados con la posición de los elementos "yo actual» y "yo ideal» en función de la condición clínica

\begin{tabular}{|c|c|c|c|c|c|c|}
\hline Variable dependiente & Condición clínica & M & DT & $F_{(1,58)}$ & $p$ & $\eta_{p}^{2}$ \\
\hline PCC & $\begin{array}{l}\text { No clínica } \\
\text { Depresiva }\end{array}$ & $\begin{array}{l}.57 \\
.28\end{array}$ & $\begin{array}{l}.18 \\
.21\end{array}$ & 31.38 & .000 & .351 \\
\hline PCD & $\begin{array}{l}\text { No clínica } \\
\text { Depresiva }\end{array}$ & $\begin{array}{l}.07 \\
.29\end{array}$ & $\begin{array}{l}.07 \\
.21\end{array}$ & 29.44 & .000 & .337 \\
\hline PCDI & $\begin{array}{l}\text { No clínica } \\
\text { Depresiva }\end{array}$ & $\begin{array}{l}.10 \\
.19\end{array}$ & $\begin{array}{l}.10 \\
.20\end{array}$ & 5.03 & .029 & .080 \\
\hline PSD & $\begin{array}{l}\text { No clínica } \\
\text { Depresiva }\end{array}$ & $\begin{array}{l}.27 \\
.24\end{array}$ & $\begin{array}{l}.14 \\
.13\end{array}$ & .65 & .422 & .011 \\
\hline
\end{tabular}

Nota. PCC (Porcentaje de Constructos Congruentes), PCD (Porcentaje de Constructos Discrepantes), PCDI (Porcentaje de Constructos Dilemáticos), PSD (Porcentaje de Constructos Sin Definir). 
Posición de los elementos «yo actual» y «yo ideal»

Para estudiar la posición de los elementos «yo actual» $\mathrm{y}$ «yo ideal» se realizó un MANOVA tomando como variables dependientes: Porcentaje de Constructos Congruentes, Porcentaje de Constructos Discrepantes, Porcentaje de Constructos Dilemáticos y Porcentaje de Categorías Indefinidas en cada rejilla. Los resultados de los contrastes multivariados fueron significativos: Lambda de Wilks $=0.575, F(3,56)$ $=13.781, p=0.000, \eta^{2}=0.425$. En la tabla 2 se muestran los estadísticos descriptivos y los resultados de las pruebas inter-sujetos.

En la figura 1 se muestra gráficamente el porcentaje de constructos congruentes, discrepantes, dilemáticos y sin definir en los grupos clínico y no clínico.
La distribución porcentual de los constructos atendiendo a la posición "yo actual» y «yo ideal» para cada condición clínica en cada virtud/fortaleza se analizó en dos tablas de contingencia. El análisis de la relación entre las tres variables mediante la prueba Chicuadrado mostró que, en ambos casos, estaban relacionadas $X^{2}(3)=176.275, p=0.000$. En la tabla 3 (virtudes) y en el apéndice B (fortalezas del carácter) se muestran los resultados ordenados de mayor a menor significación estadística.

En relación a los constructos congruentes se observan diferencias intergrupales importantes ya que en el grupo no clínico representan el 58.7\% del total de constructos frente al $27.6 \%$ de la totalidad de constructos en el grupo clínico.

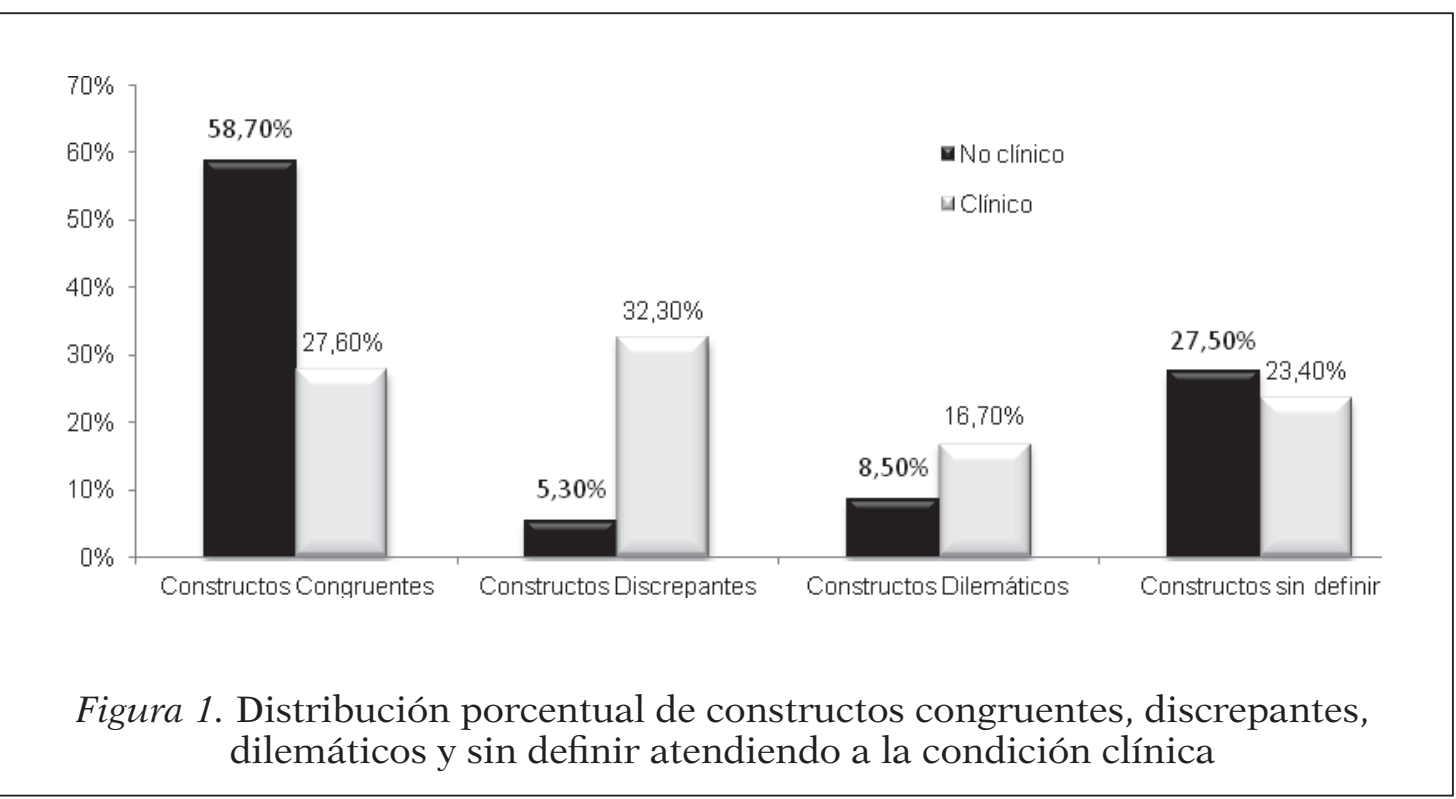


Tabla 3

Distribución porcentual, residuos tipificados corregidos y valor de Chi cuadrado y grados de libertad de la tabla de contingencia de las categorías

\begin{tabular}{|c|c|c|c|c|c|c|c|c|c|c|c|}
\hline & & \multicolumn{2}{|c|}{ Congruentes } & \multicolumn{2}{|c|}{ Discrepantes } & \multicolumn{2}{|c|}{ Dilemáticos } & \multicolumn{2}{|c|}{ Sin definir } & \multirow[t]{2}{*}{$X^{2}$} & \multirow[t]{2}{*}{$p$} \\
\hline & & NC & C & NC & C & NC & C & NC & C & & \\
\hline Coraje & $\begin{array}{r}\text { Rec. } \\
\% \\
\text { RTC }\end{array}$ & $\begin{array}{r}84 \\
56.40 \\
5.7 \\
\end{array}$ & $\begin{array}{r}21 \\
20.40 \\
-5.7 \\
\end{array}$ & $\begin{array}{r}13 \\
8.70 \\
-6.6 \\
\end{array}$ & $\begin{array}{r}46 \\
44.70 \\
6.6 \\
\end{array}$ & $\begin{array}{r}6 \\
4.00 \\
-2.8 \\
\end{array}$ & $\begin{array}{r}14 \\
13.60 \\
2.8 \\
\end{array}$ & $\begin{array}{r}46 \\
30.90 \\
1.7 \\
\end{array}$ & $\begin{array}{r}22 \\
21.40 \\
-1.7 \\
\end{array}$ & 61.583 & 0.000 \\
\hline Transcendencia & $\begin{array}{r}\text { Rec. } \\
\% \\
\text { RTC }\end{array}$ & $\begin{array}{r}32 \\
56.10 \\
4.3\end{array}$ & $\begin{array}{r}8 \\
15.70 \\
-4.3\end{array}$ & $\begin{array}{r}2 \\
3.50 \\
-6.8\end{array}$ & $\begin{array}{r}33 \\
64.70 \\
6.8\end{array}$ & $\begin{array}{r}1 \\
1.80 \\
-1.1\end{array}$ & $\begin{array}{r}3 \\
5.90 \\
1.1\end{array}$ & $\begin{array}{r}22 \\
38.60 \\
2.9\end{array}$ & $\begin{array}{r}7 \\
13.70 \\
-2.9\end{array}$ & 50.438 & 0.000 \\
\hline Humanidad & $\begin{array}{r}\text { Rec. } \\
\% \\
\text { RTC }\end{array}$ & $\begin{array}{r}107 \\
68.20 \\
5.4\end{array}$ & $\begin{array}{r}55 \\
37.40 \\
-5.4\end{array}$ & $\begin{array}{r}3 \\
1.90 \\
-5.3\end{array}$ & $\begin{array}{r}31 \\
21.10 \\
5.3\end{array}$ & $\begin{array}{r}18 \\
11.50 \\
-0.9\end{array}$ & $\begin{array}{r}22 \\
15.00 \\
0.9\end{array}$ & $\begin{array}{r}29 \\
18.50 \\
-1.7\end{array}$ & $\begin{array}{r}39 \\
26.50 \\
1.7\end{array}$ & 41.337 & 0.000 \\
\hline Moderación & $\begin{array}{r}\text { Rec. } \\
\% \\
\text { RTC }\end{array}$ & $\begin{array}{r}46 \\
47.40 \\
3.9\end{array}$ & $\begin{array}{r}21 \\
21.20 \\
-3.9\end{array}$ & $\begin{array}{r}8 \\
8.20 \\
-3\end{array}$ & $\begin{array}{r}24 \\
24.20 \\
3\end{array}$ & $\begin{array}{r}14 \\
14.40 \\
-2.2\end{array}$ & $\begin{array}{r}27 \\
27.30 \\
2.2\end{array}$ & $\begin{array}{r}29 \\
29.90 \\
0.4\end{array}$ & $\begin{array}{r}27 \\
27.30 \\
-0.4\end{array}$ & 21.504 & 0.000 \\
\hline Sabiduría & $\begin{array}{r}\text { Rec. } \\
\% \\
\text { RTC }\end{array}$ & $\begin{array}{r}48 \\
59.30 \\
3.2\end{array}$ & $\begin{array}{r}17 \\
31.50 \\
-3.2\end{array}$ & $\begin{array}{r}4 \\
4.90 \\
-3.5\end{array}$ & $\begin{array}{r}14 \\
25.90 \\
3.5\end{array}$ & $\begin{array}{r}7 \\
8.60 \\
-1.4\end{array}$ & $\begin{array}{r}9 \\
16.70 \\
1.4\end{array}$ & $\begin{array}{r}22 \\
27.20 \\
0.2\end{array}$ & $\begin{array}{r}14 \\
25.90 \\
-0.2\end{array}$ & 17.675 & 0.001 \\
\hline Justicia & $\begin{array}{r}\text { Rec. } \\
\% \\
\text { RTC }\end{array}$ & $\begin{array}{r}13 \\
61.90 \\
0.7\end{array}$ & $\begin{array}{r}7 \\
50.00 \\
-0.7\end{array}$ & $\begin{array}{r}0 \\
0.00 \\
-2.2\end{array}$ & $\begin{array}{r}3 \\
21.40 \\
2.2\end{array}$ & $\begin{array}{r}2 \\
9.50 \\
-1\end{array}$ & $\begin{array}{r}3 \\
21.40 \\
1\end{array}$ & $\begin{array}{r}6 \\
28.60 \\
1.6\end{array}$ & $\begin{array}{r}1 \\
7.10 \\
-1.6\end{array}$ & 7.47 & 0.058 \\
\hline
\end{tabular}

Nota. Rec. (recuento); \% (porcentaje de constructos dentro de cada población); RTC (residuos tipificados corregidos); NC (grupo no clínico); C (grupo clínico).

Las casillas que aparecen sombreadas en color gris corresponden al análisis de residuos tipificados corregidos estadísticamente significativos $(R T C \geq|1.96|)$.

\section{Diferenciación del sí mismo}

Con el objetivo de explorar la relación entre la condición clínica y los distintos índices de diferenciación del sí mismo, se realizó un MANOVA. Los resultados mostraron un efecto significativo de la variable independiente condición clínica: Lambda de Wi$l k s=0.650, F(3,56)=10.043, p=0.000, \eta_{p}^{2}$ $=0.350$. En la tabla 4 se muestran los estadísticos descriptivos y los resultados de las pruebas inter-sujetos.

Tabla 4

Medias, desviaciones típicas y significación estadística de los índices de la diferenciación del sí mismo en función de la condición clínica

\begin{tabular}{|c|c|c|c|c|c|c|}
\hline Variable dependiente & $\begin{array}{l}\text { Condición } \\
\text { clínica }\end{array}$ & $\mathbf{M}$ & DT & $F_{(1,58)}$ & $p$ & $\eta^{2}$ \\
\hline Correlación «yo actual»-«yo ideal» & $\begin{array}{l}\text { No clínica } \\
\text { Depresiva }\end{array}$ & $\begin{array}{l}.54 \\
-.21\end{array}$ & $\begin{array}{l}.60 \\
.52\end{array}$ & 26.55 & .000 & .314 \\
\hline Correlación «yo actual»-«otros» & $\begin{array}{l}\text { No clínica } \\
\text { Depresiva }\end{array}$ & $\begin{array}{l}.42 \\
.10\end{array}$ & $\begin{array}{l}.34 \\
.42\end{array}$ & 17.30 & .000 & .230 \\
\hline Correlación «yo ideal»-«otros» & $\begin{array}{l}\text { No clínica } \\
\text { Depresiva }\end{array}$ & $\begin{array}{l}.49 \\
.44\end{array}$ & $\begin{array}{l}.36 \\
.32\end{array}$ & .39 & .533 & .007 \\
\hline
\end{tabular}

Nota. A los coeficientes de correlación se les aplicó la transformación Z de Fisher. 


\section{Discusión}

En este trabajo se ha explorado la construcción del sí mismo en un grupo de personas con trastornos depresivos frente a un grupo no clínico evaluados mediante la Técnica de la Rejilla. Para ello, se ha estudiado en primer lugar el contenido de los constructos a través de la clasificación que Peterson y Seligman (2004) establecen sobre las virtudes y las fortalezas del carácter y, posteriormente, se ha analizado la posición entre los elementos «yo actual»y «yo ideal» con el objetivo de explorar la autodefinición del sí mismo. En un segundo lugar, se ha estudiado la diferenciación del sí mismo operativizada a través del estudio de la autoestima, el aislamiento social autopercibido y la adecuación percibida en los otros.

Con respecto al análisis del contenido de los constructos personales, el mayor número de constructos elicitados por ambos grupos se concentran en las categorías Humanidad, Coraje y Moderación, frente a las de Sabiduría, Trascendencia y Justicia. Estos resultados podrían estar indicando que las primeras tres virtudes tienen mayor relevancia en su red de significados personales. Del mismo modo, en cuanto al análisis por fortalezas, ambos grupos elicitan principalmente constructos pertenecientes a las categorías de autocontrol y autorregulación (Moderación), vitalidad y pasión por las cosas (Transcendencia) e inteligencia emocional, personal y social (Humanidad ). Sin embargo, en este caso se encuentran diferencias significativas entre los grupos: las personas del grupo no clínico elicitan mayor número de constructos en la categoría vitalidad y pasión por las cosas, mientras que las personas del grupo clínico expresan mayoritariamente significados pertenecientes a las categorías autocontrol y autorregulación e inteligencia emocional, personal y social. El autocontrol y la autorregulación (i.e., nerviosa vs. tranquila, histérica vs. tranquila; con prontos vs. sin prontos) podría relacionarse con el desajuste psicológico que experimentan las personas con depresión y la dificultad que presentan a la hora de comprender y regular su estado afectivo negativo (Nolen-Hoeksema, 1991, citado en Ramos, Fernández-Berrocal y Extremera, 2003). Por otro lado, el alto porcentaje de constructos englobados dentro de la categoría de inteligencia emocional, personal y social enfatiza la importancia que para las personas con depresión tienen los afectos y emociones, algo que también ha sido valorado en otros estudios en los que se señala la primacía de constructos sobre afectividad en pacientes depresivos (Feixas et al., 2002, 2009; González-Arranz, 2011). Además de la exploración del contenido de los significados mediante su asignación a un sistema categorial, en este trabajo se ha investigado en qué medida los sujetos se definen a sí mismos de forma similar o diferente a lo que ellos consideran su ideal. Teniendo en cuenta esta idea, se han encontrado diferencias estadísticamente significativas entre los dos grupos estudiados. Así, el grupo clínico presenta un menor porcentaje de constructos congruentes y un mayor número de constructos discrepantes y dilemáticos que el grupo no clínico. Estos resultados se sitúan en la línea de los encontrados en otras investigaciones (Feixas y Saúl, 2004; Saúl, 2005) en las que se evidencia que las personas con depresión no se encuentran a gusto consigo mismas y desean cambiar.

En cuanto a los constructos discrepantes, las diferencias grupales son notables y atañen fundamentalmente a las categorías de esperan$z a$, optimismo y proyección hacia el futuro (Transcendencia), perseverancia $y$ diligencia (Coraje), autocontrol o autorregulación (Moderación), inteligencia emocional, personal y social (Humanidad) y vitalidad y pasión por las cosas (Coraje), en el sentido de que las personas con depresión tienden a situarse en el polo contrario a su «yo ideal» en estas categorías (ver Apéndice 2). En relación a la categoría esperanza, optimismo y proyección hacia el futuro, las personas con depresión se sienten tristes desesperanzadas, pesimistas y esta visión dista mucho de su ideal, que está emplazado en ser una persona optimista. Estos resultados se sitúan en consonancia con otros estudios que informan de una relación negativa entre el optimismo y la depresión (Marrero y Carballeira, 2010) y positiva entre el optimismo y el bienestar personal (Vázquez, Hervás, Rahona y Gómez, 2009). De igual forma, en la categoría de vitalidad y pasión por las cosas, las personas 
con depresión son conscientes de su falta de energía, resultados coincidentes con autores como Beck et al. (1979/2000) quienes afirman que es característico en las personas con depresión la presencia de incapacidad para sentir placer, la ausencia de entusiasmo antes nuevos y la disminución de sensaciones gratificantes. Por último, con respecto a la perseverancia y diligencia, la falta de energía coincide con las ideas de Landfield (1971) quien afirma que la «disminución de la fuerza» es la categoría que mejor define a las personas con depresión.

Con respecto a los constructos dilemáticos, las diferencias encontradas entre los grupos clínico y no clínico se circunscriben a los constructos categorizados en las virtudes de Coraje y Moderación. El hecho de que la puntuación del «yo ideal» no se sitúe en ninguno de los dos polos, esto es, en una puntuación media, se ha interpretado como que dicho significado no le ofrece al sujeto un curso claro de acción (Feixas y Saúl, 2004), o como un proceso de constricción (Winter, Bell y Watson, 2010). En todo caso, el corolario de elección de Kelly afirma que una persona elige aquel polo del constructo que supone una mayor elaboración para su sistema de anticipación; pero podría ocurrir que cualquiera de los dos polos del constructo no sea deseado por el sujeto, siguiendo la máxima aristotélica de in medio virtus. En este sentido, esta explicación podría ser bastante evidente en el caso de los constructos dilemáticos clasificados dentro de la virtud de moderación, dónde la posición intermedia sí tendría sentido en la mayoría de los casos. Así, son las categorías de autocontrol o autorregulación las que recogen el mayor número de puntuaciones medias en el «yo ideal» (e.g., nerviosa vs. pasiva).

No obstante, en otros casos, definir al «yo ideal» en uno de los polos sería fundamental para marcar la línea de acción del sujeto. De este modo, los constructos dilemáticos clasificados dentro de la virtud de Coraje darían pistas de que la persona no presenta construcciones viables de significado y podríamos estar ante situaciones de bloqueo y falta de movilidad. El Coraje sería determinante en una persona con depresión para romper el bloqueo del «no movimiento» al que está sometido. Que la persona no sepa situar a su ideal, por ejemplo, en el constructo "no muestra interés vs. se preocupa por las cosas» sería un verdadero conflicto cognitivo para ella y una posible explicación de la resistencia al cambio, fruto del dilema al que se ve sometida (Feixas y Saúl, 2004; Sánchez y Feixas, 2001). En cualquier caso, y tal como indican Feixas et al. (2009), las puntuaciones medias en el "yo ideal» e incluso del "yo actual», están abiertas a la discusión, a la crítica y a una mayor elaboración.

Los resultados encontrados en cuanto a la autodefinición en las personas con depresión nos llevan a dos consideraciones. Por un lado, estas personas resaltan las discrepancias con respecto a lo que les gustaría ser, evidenciando la carga de sufrimiento inherente a ello, y por otro lado, la fijación en aspectos negativos les impide ver sus posibles fortalezas de carácter. De manera más específica, la psicología positiva clínica plantea el desarrollo y amplitud de las fortalezas de carácter como el aspecto esencialmente ligado a la prevención de recaídas y al mantenimiento de la salud mental (Moeenizadeh y Salagame, 2010), entendida en sentido amplio.

El segundo de los objetivos de este trabajo fue estudiar cómo las personas de ambos grupos construyen su mundo de significados mediante diversos índices cognitivos sobre la base de la diferenciación entre el sí mismo con el ideal y con los demás; a saber, el análisis de la discrepancia entre el "yo actual» - «yo ideal» (como una medida de autoestima), la diferenciación "yo actual - otros» (como una medida de aislamiento social autopercibido) y la diferenciación "ideal - otros» (como una medida de la adecuación percibida en los otros) (Feixas y Cornejo, 1996). Con respecto a la medida de autoestima (correlación «yo actual» - «yo ideal») en los pacientes con depresión, se ha obtenido una correlación negativa, lo que sugiere una construcción negativa del yo y una baja autoestima (Fernandes y Gonçalves, 1997; Feixas y Cornejo, 1996). Además, estos sujetos se construyen a sí mismos como diferentes con respecto a su entorno social puesto que, aunque la correlación entre "yo actual» $\mathrm{y}$ «otros» es positiva, no alcanza la significación estadística. Esta medida es un indi- 
cador de la soledad del sujeto y se interpreta como una construcción del yo aislado interpersonalmente. Además, cabría añadir que la diferencia encontrada en el número de elementos elicitados es menor en los sujetos depresivos, lo cual es indicativo de una menor red de relaciones interpersonales. Estos datos son coincidentes con investigaciones en las que se constata que lo que caracteriza a las personas con depresión es una autoestima significativamente menor y una percepción más negativa de sí mismos y de los demás (Axford y Jerrom, 1986; Hewstone, Hooper y Miller, 1981). En cuanto a la correlación "yo ideal» - «otros», los datos indican que los "otros» se acercan al «yo ideal», proporcionando un índice de buena adecuación percibida en los otros. No se encuentran diferencias estadísticamente significativas entre ambos grupos y la correlación, en ambos casos, es positiva. De esta manera, surge un sesgo en la información de uno mismo hacia lo negativo y otro sesgo, esta vez positivo, en relación a los otros, "yo no me parezco al ideal que tengo de mi, no me parezco a los otros pero los demás sí se parecen a mi ideal». El grupo de Feixas (Feixas y Cornejo, 1996; Feixas et al., 2008) establece una serie de perfiles tentativos en la construcción del sí mismo de acuerdo a estos tres índices, considerando el Perfil de Aislamiento en el caso de una correlación negativa entre «yo actual» - «yo ideal» y «yo actual» - «otros»y, una correlación positiva entre «yo ideal» - «otros». Este perfil, es característico de personas con depresión tal y como predice la teoría cognitiva de Beck (Beck, Rush, Shaw y Emery, 1983) y que concuerda con los datos arrojados en otros estudios dentro del marco de la PCP (Axford y Jerrom, 1986; Feixas et al., 2008; Hewstone,
Hooper y Miller, 1981; Oliver y McGee, 1982; Space y Cromwell, 1980).

De cara a futuras investigaciones, sería interesante profundizar en el estudio de la posición de los elementos "yo actual» y "yo ideal» en el sentido de identificar y evaluar el cambio en los patrones de los constructos personales durante la terapia o en qué medida los constructos se modifican en función de la evolución de la depresión.

Una limitación del presente trabajo es el tamaño muestral. Al tratarse de una muestra pequeña, la validez externa se ve reducida y, por tanto, resulta aventurado realizar afirmaciones decisivas sobre los resultados obtenidos. En este sentido, sería deseable la realización de nuevos estudios con muestras mayores que nos aporten nuevos datos para corroborar, en su caso, los resultados obtenidos en esta investigación. No obstante, en estas nuevas investigaciones sería recomendable el análisis y control apriorístico, en su caso, de algunas variables sociodemográficas y clínicas como la edad, el nivel de estudios/cultural o posibles trastornos de ansiedad co-mórbidos, que pudieran estar condicionando los resultados obtenidos.

Finalmente, consideramos que la TR es instrumento valioso para conocer las virtudes y fortalezas del carácter que desempeñan un papel relevante en la construcción del sí mismo en las personas con depresión. Este conocimiento, en el marco terapéutico, puede ayudar a identificar aquellas categorías críticas para la persona y, en línea con lo planteado por Guidano (1999), orientar el "cambio» entendido como construcción de un equilibrio progresivo que se va logrando como resultado de una reorganización del significado personal. 


\section{Referencias}

American Psychiatric Association (2000). Diagnostic and Statistc Manual of Mental Disorders (4. ${ }^{a} \mathrm{Ed}$. Rev.). Barcelona, España: Masson

Avia, M. D. y Vázquez, C. (1998). Optimismo inteligente. Psicología de las emociones positivas [Intelligent Optimism. Psychology of Positive Emotions]. Madrid, España: Alianza.

Axford, S. y Jerrom, D. W. A. (1986). Self-esteem in depression: A controlled repertory grid investigation. British Journal of Medical Psychology, 59, 61-68.

Beck, A. T. (1967). Depression: Clinical, experimental, and theoretical aspects. Nueva York, NY: Harper \& Row.

Beck, A. T. (1976). Cognitive therapy and the emotional Disorders. Nueva York, NY: International University Press.

Beck, A. T., Rush, A. J., Shaw, B. F. y Emery, G. (1983/2000). Terapia cognitiva de la depresión [Cognitive Therapy of Depression]. Bilbao, España: Desclée De Brouwer.

Botella, L. y Feixas, G. (1998). Teoría de los constructos personales: Aplicaciones a la práctica psicológica [Personal construct theory: Applications to psychological practice]. Barcelona, España: Laertes.

Carr, A. (2004). Positive Psychology. The science of happiness and human strengths. Nueva York, NY: Brunner-Routledge.

Chico, E. (2002). Optimismo disposicional como predictor de estrategias de afrontamiento [Dispositional optimism as a predictor of strategies coping]. Psicothema, 14, 544-550.

Chico, E. y Ferrando, P. J. (2008). Variables cognitivas y afectivas como predictoras de satisfacción en la vida [Cognitive and affective variables as predictors of life satisfaction]. Psicothema, 20, 408-412.

De Bonis, M. (1998). Thinking and Depression: Structure in Content. En J. William F. Flack y J. D. Laird (Eds.), Emotions in Psychopathology: Theory and Research (pp. 185-199). New York, US: Oxford University Press US.

Derogatis, L. (1994). SCL-90-R. Symptom Checklist90-R. Administration, Scoring and Procedures Manual. Minneapolis: National Computer System.
Extremera, N., Fernández-Berrocal, P. y Salovey, P. (2006). Spanish Version of the Mayer-SaloveyCaruso Emotional Intelligence Test (MSCEIT) Version 2.0: Reliabilities, Age, and Gender Differences. Psicothema, 18, 42-48.

Feixas, G. y Cornejo, J. M. (2002). RECORD v. 4.0: Análisis de correspondencias de constructos personales [programa informático] [Gridcor v. 4.0: Correspondence Analysis of personal constructs]. Barcelona, España: Psimedia. Recuperado de www.terapiacognitiva.net/record.

Feixas, G. y Saúl, L. A. (2004). The Multi-Center Dilemma Project: an Investigation on the Role of Cognitive Conflicts in Health. The Spanish Journal of Psychology, 7(1), 69-78.

Feixas, G. y Botella, L. (2003). Las técnicas subjetivas [Subjective techniques]. En C. Moreno (Ed.), Evaluación psicológica. Concepto, proceso y su aplicación en las áreas del desarrollo y de la inteligencia (pp. 307-342). Madrid, España: Sanz y Torres.

Feixas, G. y Cornejo, J. M. (1996). Manual de la Técnica de Rejilla mediante el programa RECORD V. 2.0 [Manual of the Repertory Grid Technique using Gridcor v. 2.0]. Barcelona, España: Paidós Ibérica.

Feixas, G., Erazo-Caicedo, M. I., Harter, S. L. y Bach, L. (2008). Construction of self and others in unipolar depressive disorders: A study using repertory grid technique. Cognitive Therapy and Research, 32, 386-400. doi:10.1007/s10608-0079149-7

Feixas, G., Geldschläger, H., Carmona, M. y Garzón, B. (2002). Sistema de Categorías de Contenido para codificar constructos personales [Content category system for codifying personal constructs]. Revista de Psicología General y Aplicada, 55(3), 337-348.

Feixas, G., Saúl, L. A. y Avila-Espada, A. (2009). Viewing cognitive conflicts as dilemmas: implications for mental health. Journal of Constructivist Psychology, 22, 141-169. doi:10.1080/10720530802675755

Fernandes, E. y Gonçalves, O. (1999). Constructos persoais sobre o self em mudanca [Personal constructs of the changing self]. Revista Argentina de Clínica Psicológica, 8(2), 145-157. 
Fernández-Berrocal, P., Extremera, N. y Ramos, N. (2004). Validity and reliability of the Spanish modified version of the Trait Meta-Mood Scale. Psychological Reports, 94, 751-755.

González Arranz, B. (2011). El cambio del contenido de los constructos personales a lo largo del proceso terapéutico (Tesis Doctoral) [The change of personal construct contents along the therapeutic process]. Salamanca: Ediciones Universidad de Salamanca.

González de Rivera, J. L., de las Cuevas, M., Rodríguez, M. y Rodríguez, F. (2002). SCL-90-R. Cuestionario de 90 sintomas [SCL-90-R. Questionnaire of 90 Symptoms]. Madrid, España: TEA.

Hanieh, E. y Walker, B. M. (2007). Photography as a measure of constricted construing: The experience of depression through a camera. Journal of Constructivist Psychology, 20(2), 183-200.

Hervás, G. (2008). Adaptación al castellano de un instrumento para evaluar es estilo rumiativo: La Escala de Respuestas Rumiativas [Spanish adaptation of an instrument to assess ruminative style: ruminative responses scale]. Revista de Psicopatología y Psicología Clínica, 13, 111-121.

Hewstone, M., Hooper, D. y Miller, K. (1981). Psychological change in neurotic depression: A repertory grid and personal construct theory approach. British Journal of Psychiatry, 139, 47-51.

Hinze, T., Doster, J. y Joe, V. C. (1997). The relationship of conservatism and cognitive-complexity. Personality and Individual Differences, 22(2), 297-298. doi: 10.1016/s0191-8869(96)00171-7.

Honess, T. (1976). Cognitive Complexity and Social Prediction. The British Journal of Social and Clinical Psychology, 15(1), 23-31.

Kelly, G. A. (1955/1991). The Psychology of Personal Constructs. A Theory of Personality (Vol. 1). New York: Norton.

Landfield, A. W. (1971). Personal construct systems in psychotherapy. Chicago: Rand McNally.

Livesay, J. R. (1984). Cognitive complexity-simplicity and inconsistent interpersonal judgment in thought-disordered schizophrenia. Psychological Reports, 54(3), 759-768.

Marcet, C. (1985). La dimensión "ComplejidadSimplicidad cognitiva»: principales índices de medida [The cognitive complexity-simplicity dimension: Principal indexes of measurement]. Cuadernos de Psicología, I, 79-97.

Marrero, R. y Carballeira, M. (2010). El papel del optimismo y del apoyo social en el bienestar subjetivo [The role of optimism and social support on subjective well-being]. Salud Mental, 33, 39-46.

Maruta, T., Colliga, R. C., Malinchoc, M. y Offord, K. P. (2000). Optimists vs pessimists: survival rate among medical patients over a 30-year period. Mayo Clinic Proceedings, 75(2), 140-143.

Mayer, J. D., Salovey, P. y Caruso, D. R. (2002). Mayer-Salovey-Caruso Emotional Intelligence Test (MSCEIT) item booklet. Toronto, Ontario, Canada: MHS Publishers.

Moeenizadeh, M. y Salagame, K.K.K. (2010). WellBeing (WBT) for DepressionTherapy. International Journal of Psychological Studies, 2, 107-115.

Montesano, A., Feixas, G. y Varlotta, N. (2009). Análisis de contenido de constructos personales en la depresión. Salud Mental, 32, 371-379.

Nolen-Hoeksema, S. (1991) Responses to Depression and Their Effects on the Duration of Depressive Episodes. Journal of Abnormal Psychology, 100, 569-582.

Nolen-Hoeksema, S. (2000) The role of rumination in depressive disorders and mixed anxiety/depressive symptoms. Journal of Abnormal Psychology, 109, 504-511.

Nolen-Hoeksema, S. y Morrow, J. (1991). A prospective study of depression and posttraumatic stress symptoms after a natural disaster: The 1989 Loma Prieta earthquake.Journal of Personality and Social Psychology, 61, 115-121.

Oliver, J. M. y McGee, J. (1982). Cognition as a function of depression in a student population: Content and complexity of cognitions. Cognitive Therapy and Research, 6, 275-286. doi:10.1007/ BF01173576.

Peterson, Ch. y Seligman, M. E. P. (2001). VIA Inventory of Strengths (VIA-IS). Recuperado de WorldWideWeb: http://www.positivepsychology. org/viastrenghtsinventory.htm.

Peterson, Ch. y Seligman, M. E. P. (2004). Character strengths and virtues: a handbook and classification. New York: Oxford University Press. 
Ramos, N., Fernández-Berrocal, P. y Extremera, N. (2003). Inteligencia emocional en situaciones de estrés agudo. En A. Jiménez (Ed.), Procesos psicológicos e intervención: investigaciones en curso [Emotional intelligence in situations of acute stress] (pp. 26-30). Málaga, España: Facultad de Psicología.

Ryan, R. M. y Deci, E. L. (2001). On happiness and human potentials: a review of research on hedonic and eudaimonic well-being. Annual Review of Pyschology, 52, 141-166.

Salovey, P., Mayer, J. D., Goldman, S. L., Turvey, C. y Palfai, T. P. (1995). Emotional attention, clarity, and repair: exploring emotional intelligence using the Trait Meta-Mood Scale. En J. W. Pennebaker (Ed.), Emotion, Disclosure, and Health (pp. 125-154). Washington: American Psychological Association.

Sánchez, V. y Feixas, G. (2001). La «resistencia» en la psicología de los constructos personales [The «resistance» in the psychology of personal constructs]. Revista de Psicoterapia, 12(46/47), 133-162.

Sanz, J. (1991). Hacia un modelo de depresión desde la psicología de los constructos personales [Towards a model of depression from the psychology of personal constructs]. Boletin de Psicología, 33, 7-40.

Sanz, J. (1992). Constructos personales y sintomatología depresiva: un estudio longitudinal [Personal constructs and depressive symptoms: a longitudinal study]. Revista de Psicología General y Aplicada, 45, 403-411.

Sanz, J. y Vázquez, C. (2008) Trastornos del estado del estado de ánimo: Teorías Psicológicas. En A. Belloch, B. Sandín y F. Ramos (Eds.), Manual de psicopatología (Vol. 2) [Manual Psychopathology] (pp. 233-270). Madrid, España: McGraw Hill.
Saúl, L. A. (2005). El papel de los conflictos cognitivos en la salud mental: implicaciones para el cambio terapéutico [Role of Cognitive Conflict in Mental Health: Implications to Therapeutic Change] (Tesis Doctoral). Universidad de Salamanca, Salamanca, España.

Seligman, M. E. P. (2003). La auténtica Felicidad [Authentic Happiness]. Barcelona, España: B.S.A.

Senra, J., Feixas, G. y Fernandes, E. (2005). Manual de intervención en dilemas implicativos [Manual of intervention in implicative dilemmas]. Revista de Psicoterapia, 63-64, 179-201.

Space, L. G. y Cromwell, R. L. (1980). Personal constructs among depressed patients. The Journal of Nervous and Mental Disease, 168, 150-158. doi:10.1097/00005053-198003000-00005

Tobacyk, J. (1983). Cognitive complexity and paranormal beliefs. Psychological Reports, 52(1), 101-102.

Vázquez, C., Hervás, G., Hernangómez, L. y Romero, N. (2010). Modelos cognitivos de la depresión: Una revisión tras 30 años de investigación [Cognitive models of depression: a review after 30 years of research]. Psicología Conductual, 18, 139-165.

Vázquez, C., Hervás, G., Rahoha, J. J. y Gómez, D. (2009). Bienestar psicológico y salud: Aportaciones desde la Psicología Positiva [Psychological well-being and health: Contributions from Positive Psychology]. Anuario de Psicología Clínica y de la Salud, 5, 15-28.

Winter, D. A., Bell, R. C. y Watson, S. (2010). Midpoint Ratings on Personal Constructs: Constriction or the Middle Way? Journal of Constructivist Psychology, 23(4), 337-356. http://dx.doi.or $\mathrm{g} / 10.1080 / 10720537.2010 .502428$ 


\section{APÉNDICE A}

Virtudes y fortalezas del character (Peterson y Seligman, 2001, 2004)

\begin{tabular}{ll}
\hline Sabiduría & Coraje \\
\hline S1: Curiosidad, Interés por el mundo. & C6: valentía \\
S2: amor por el conocimiento y el aprendizaje & C7: perseverancia y diligencia \\
S3: juicio, pensamiento critico, mentalidad abierta & C8: integridad, honestidad y autenticidad \\
S4: ingenio, originalidad e inteligencia práctica & C9: vitalidad y pasión por las cosas \\
S5: perspectiva & \\
\hline Humanidad & Justicia \\
\hline H10: amor, apego, capacidad de amar y ser & J13: ciudadanía, civismo, lealtad y trabajo \\
amado & en equipo \\
H11: simpatía, amabilidad y generosidad & J14: sentido de la justicia, equidad \\
H12: inteligencia emocional, personal y social & J15: liderazgo \\
\hline Moderación & Trascendencia \\
\hline M16: capacidad de perdonar, misericordia & T20: apreciación de la belleza y la \\
M17: modestia o humildad & excelencia y la capacidad de asombro \\
M18: prudencia, discreción o cautela & T21: gratitud \\
M19: autocontrol o autorregulación & T22: esperanza, optimismo, proyección \\
& hacia el futuro \\
& T23: sentido del humor \\
& T24: espiritualidad, fe y sentido religioso
\end{tabular}




\section{APÉNDICE B}

Recuento, distribución porcentual, residuos tipificados corregidos y valor de Chi cuadrado y grados de libertad de la tabla de contingencia de las categorías

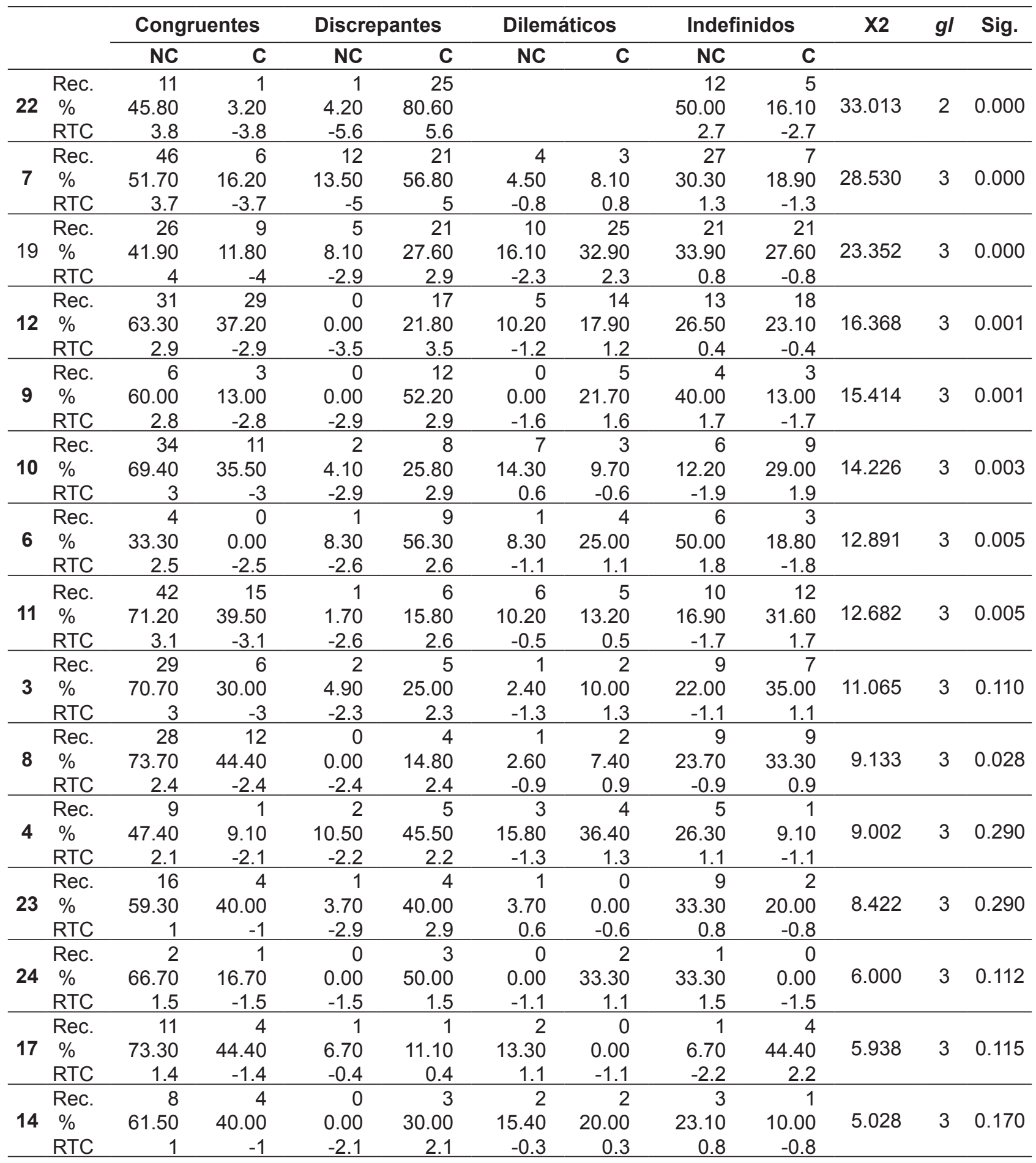




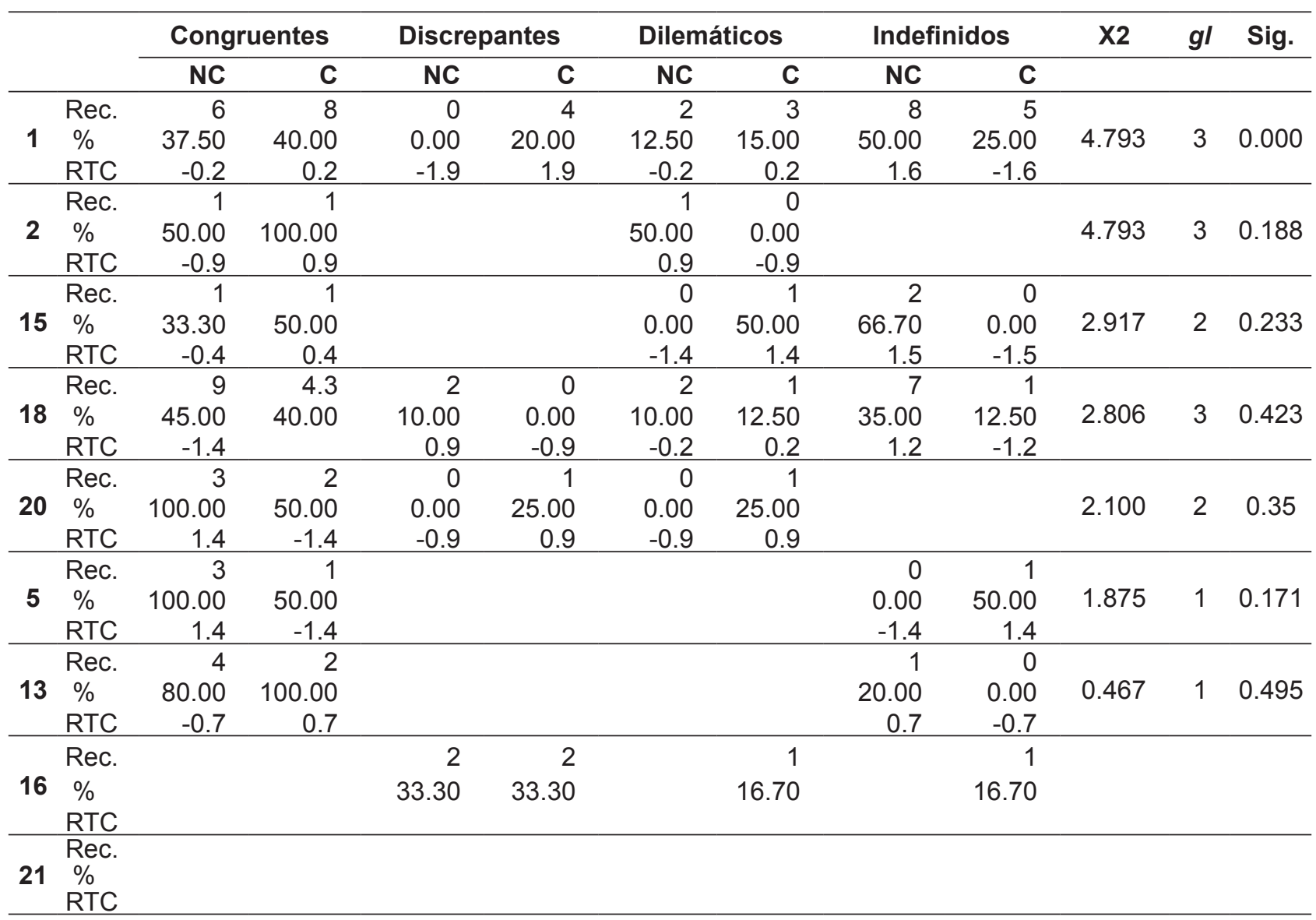

Nota. Rec. (recuento), \% (frecuencia dentro de cada fila para cada condición clínica), RTC (Residuos Tipificados Corregidos), 1 (curiosidad, interés por el mundo), 2 (amor por el conocimiento y el aprendizaje), 3 (juicio, pensamiento crítico, mentalidad abierta), 4 (ingenio, originalidad e inteligencia práctica), 5 (perspectiva), $\mathbf{6}$ (valentía), $\mathbf{7}$ (perseverancia y diligencia), 8 (integridad, honestidad y autenticidad), 9 (vitalidad por las cosas), 10 (amor, apego, capacidad de amar y ser amado), 11 (simpatía, amabilidad y generosidad), $\mathbf{1 2}$ (inteligencia emocional, personal y social), $\mathbf{1 3}$ (ciudadanía, civismo, lealtad y trabajo en equipo), 14 (sentido de la justicia, equidad), 15 (liderazgo), 16 (capacidad de perdonar, misericordia), 17 (modestia o humildad), 18 (prudencia, discreción o cautela), 19 (autocontrol o autorregulación), 20 (apreciación de la belleza y la excelencia, capacidad de asombro), 21 (gratitud), 22 (esperanza, optimismo, proyección hacia el futuro), 23 (sentido del humor), 24 (espiritualidad, fe y sentido religioso). 